\title{
A BOUND FOR ZEROS OF SOLUTIONS TO A HIGHER ORDER NON-HOMOGENEOUS ODE WITH POLYNOMIAL COEFFICIENTS
}

\author{
MiCHAEL GIL'
}

Abstract. Let $P_{k}(z)(k=1,2, \ldots, n)$ and $G(z)$ be polynomials with complex in general coefficients. The paper deals with the higher order differential equation

$$
v^{(n)}(z)+P_{1}(z) v^{(n-1)}(z)+\ldots+P_{n}(z) v(z)=G(z) .
$$

We derive estimates for the sums of the zeros of solutions to this equation. These estimates give us bounds for the function counting the zeros of solutions and information about the zero-free domain. Some other applications are also discussed.

Mathematics subject classification (2020): 34C10, 34A30.

Keywords and phrases: Ordinary differential equations, linear equations, zeros of solutions.

\section{REFERENCES}

[1] N. ANGHEL, Stieltjes-Calogero-Gil' relations associated to entire functions of finite order, Journal of Mathematical Physics, 51 (5), (2010) 251-262.

[2] N. ANGHEL, Entire functions of finite order as solutions to certain complex differential equations, Proc. Amer. Math. Soc., 140, (2012) 2319-2332.

[3] T. B. CAO AND H. X. YI, On the complex oscillation theory of linear differential equations with analytic coefficients in the unit disc, Acta Math. Sci., 28A (6), (2008) 1046-1057.

[4] T. B. CAO, K. LIU AND H. Y. XU, Bounds for the sums of zeros of solutions of $u(m)=P(z) u$ where $P$ is a polynomial, Electron. J. Qual. Theory Differ. Equ. 60, (2011) 10 pp.

[5] A. Eremenko And S. Merenkov, Nevanlinna functions with real zeros, Illinois J. Math. 49, no. 4, (2005) 1093-1110.

[6] L. Y. GAO, On the growth of solutions of higher-order algebraic differential equations, Acta Math. Scientia (B), 22 (4), (2002) 459-465.

[7] M. I. GIL', Localization and Perturbation of Zeros of Entire Functions, CRC Press, Taylor and Francis Group, New York, 2010.

[8] M. I. GIL', Bounds for zeros of solutions of second order differential equations with polynomial coefficients, Results Math., 59, (2011) 115-124.

[9] M. I. GIL', Bounds for products of zeros of solutions to nonhomogeneous ODE with polynomial coefficients, Int. J. Differ. Equ. Art. ID 690519, (2015) 6 pp.

[10] M. I. GIL, Sums of zeros of solutions to non-homogeneous ODE with polynomial coefficients, J. Math. Anal. Appl. 421, no. 2, (2015) 1917-1924.

[11] M. I. GIL', Inequalities for zeros of solutions to second order ODE with one singular point, Differ. Equ. Appl. 8, no. 1, (2016) 69-76.

[12] I. C. Gohberg, S. Goldberg, And N. Krupnik, Traces and Determinants of Linear Operators, Birkhäuser Verlag, Basel, 2000.

[13] C. Z. HuAng, Real zeros of solutions of second order linear differential equations, Kodai Math. J., 14, (1991) 113-122.

[14] I. Laine, Nevanlinna Theory and Complex Differential Equations, Walter de Gruyter Berlin, 1993.

[15] Z. Latreuch, B. Belaidi, AND A. El Farissi, Complex oscillation of differential polynomials in the unit disc, Periodica Mathematica Hungarica, 66 (1), (2013) 45-60. 
[16] C. H. Lin, Y. SiBUYA AND T. TABARA, Zeros of solutions of a second order linear differential equation with polynomial coefficients. Funkc. Ekvacioj, 36, (1993) no. 2, 375-384.

[17] G. M. Muminov, On the zeros of solutions of the differential equation $\omega^{(2 m)}+p(z) \omega=0$, Demonstr. Math., 35, no. 1, (2002) 41-48.

[18] J. TU, AND Z. X. CHEN, Zeros of solutions of certain second order linear differential equation, J. Math. Anal. Appl. 332, no. 1 (2007) 279-291. 